\title{
Electron Annealing in Aluminum Interconnects Observed Using In Situ TEM Technique
}

Degang Xie ${ }^{1}$, Mona $\mathrm{Li}^{2}$, Zhiwei Shan ${ }^{3}$

1. Center for Advancing Materials Performance from the Nanoscale (CAMP-Nano)

2. Hysitron Applied Research Center in China (HARCC)

3. State Key Laboratory for Mechanical Behavior of Materials, Xi'an Jiaotong University, Xi'an 710049, PR China

Interconnect is a thin wire of copper or aluminum alloy which makes electrical contact between devices, typically on Si substrate. Electro-migration is the phenomenon that metallic atoms are transported by electron wind due to high electrical current density in the metal line. Ever since Blech [1] reported a relationship between interconnect length and the rate of electromigration, induced drift, other factors like crystallographic texture [2], grain size and its distribution [3] and grain boundary structures [4] have been extensively documented to have a major impact on electron-migration induced plasticity. The continuous scaling down in the dimensions of typical integrated circuits leads to increasing electric current density in interconnects lines. Consequently, understanding the exact mechanism and evolution of electrical current effect on interconnect has become critical than ever for designing reliable devices.

When interconnects' dimension shrinks into submiro- and even nano-meter range, it will becomd electron transparent inside transmission electron microscope. Consequently, in situ TEM wil become the best tool for studying the microstructure evolution of interconnects under the effect of electrical current. In this work, we developed a novel method for preparing samples that can be studied in situ inside a TEM under the effect of electrical current. Figure 1 is the schematic for the sample preparation. By employing a Hysitron PI95 ECR type holder, we found that the defects density inside the Al grains can be reduced dramatically by the applied current (Figure 2) which we termed as electron annealing [5]. This phenomenon is possible to be used to heal those defected material in integrated circuits and in MEMS devices.

\section{References}

[1] I. A. Blech, Journal of Applied Physics, 47(1976), 1203-1208.

[2] P. C. Wang, G. S. Cargill, I. C. Noyan, C. K. Hu, Applied Physics Letters, 72(1998), 1296-1298.

[3] J. Cho, C. V. Thompson, Applied Physics Letters, 54(1989), 2577-2579.

[4] K. C. Chen, W. W. Wu, C. N. Liao, L. J. Chen, K. N. Tu, Science, 321(2008), 1066-1069; bC. M. Tan, A. Roy, Materials Science \& Engineering R-Reports, 58(2007), 3-75.

[5] This work was supported by the grants from NSFC (50925104) and 973 Program of China (2010CB631003). The authors thank A. M. Minor from the National Center for Electron Microscope, Lawrence Berkeley National Laboratory, for sample preparation, useful discussion, and support from Hysitron. 


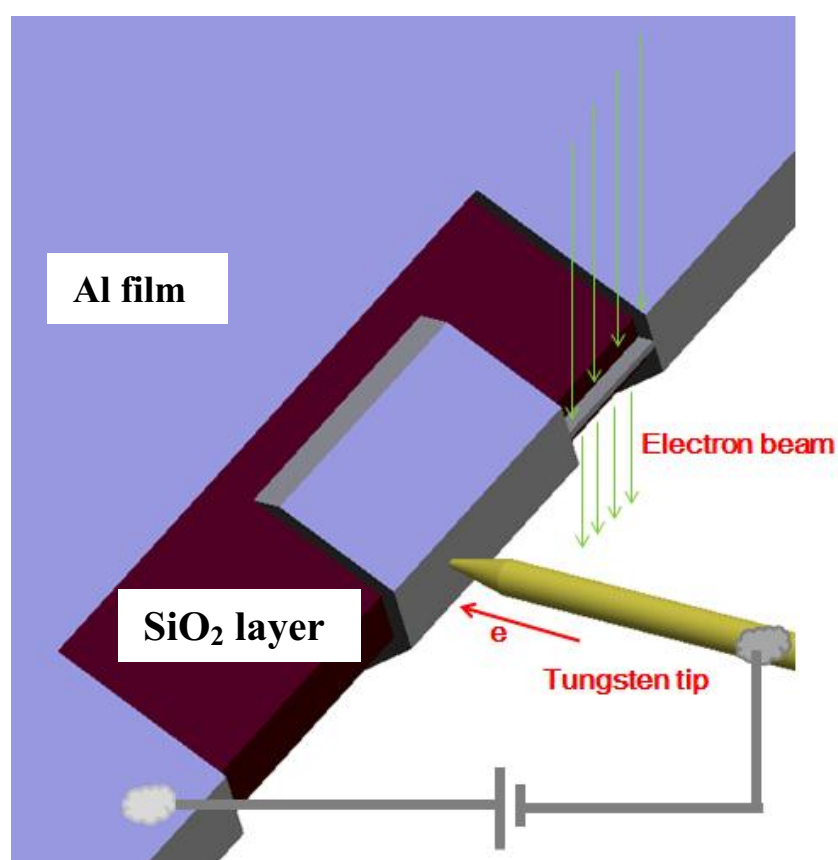

Figure 1. Schematic of the experimental setup. The sample was made from a boron doped silicon wedge with $\mathrm{SiO}_{2}$ layer and $\mathrm{Al}$ coating. FIB was used to pattern a peninsula-like sample at the top of the silicon wedge. High-density current was applied by touching the peninsula with a movable Tungsten tip and applying a electric bias between the "peninsula head" and the "mainland".

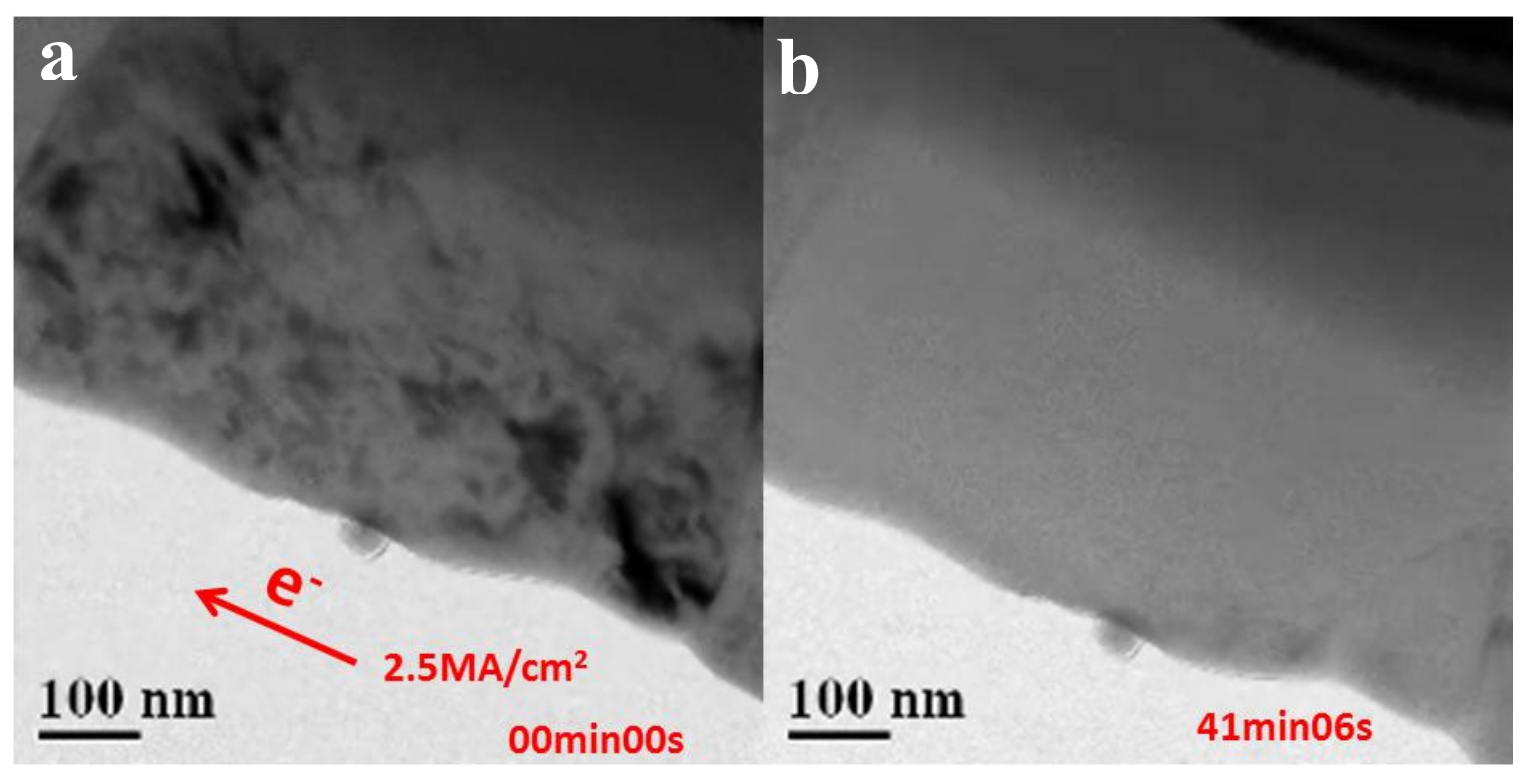

Figure 2. Bright field TEM images of a Al grain before ( a) and after (b) the applied electrical current. The defects density decreased dramatically after the treatment with electron current, a phenomena we have termed as electrical annealing. 